\title{
The Beijing Astronomical Observatory Supernova Survey
}

\author{
Yulei Qiu \& Jingyao Hu
}

Beijing Astronomical Observatory, Chinese Academy of Sciences A20, Datun Rd., Chaoyang District, Beijing 100012, China qiuyl@nova.bao.ac.cn, hjy@class1.bao.ac.cn

Weidong Li

Department of Astronomy, University of California, Berkeley, CA 94720-3411weidong@urania.berkeley.edu

\begin{abstract}
We present here the Beijing Astronomical Observatory Supernova Survey (BAOSS). The motivation and the strategy of the survey are briefly discussed, and the hardware and the software of the system are described. BAOSS started in 1996 and has discovered 40 supernovae (SNe) in four years, thus playing an important role in the search for nearby supernovae. The results of several well-observed $\mathrm{SNe}$ discovered by BAOSS, SNe 1996W, 1996cb, 1997br, and 1998S, are reported.
\end{abstract}

\section{Introduction}

After the explosions of SN 1987A in the Large Magellanic Cloud and SN 1993J in M81, there has been an increasing interest in the observation and study of $\mathrm{SNe}$. The wide-spread use of CCD detectors makes finding SNe relatively easy and the total number of SNe discovered has doubled in the last decade. A great number of photometric and spectroscopic observations have been published. As a result, the physics of supernovae is better understood than ever before. It is now widely accepted that $\mathrm{SNe}$ can be divided into several types, i.e., Type Ia, $\mathrm{II}, \mathrm{Ib}$, and Ic. The Type Ia SNe (SNe Ia), due to their relative homogeneous brightness, are used as distance indicators for cosmology, and exciting results have been obtained on the expansion history of the Universe from studies of high red-shift SNe Ia (e.g., Schmidt et al 1998; Perlmutter et al 1999). Some SNe may be correlated with Gamma Ray Bursts (Wheeler et al 2000).

The idea of conducting a supernova survey with the $60 \mathrm{~cm}$ telescope at the Xinglong Station of Beijing Astronomical Observatory (BAO) was first proposed by one of us $(\mathrm{Hu})$ in 1994, after the successful observations and analysis of SN 1993J (Wang \& Hu 1994) at BAO. The motivation was to systematically discover supernovae in nearby galaxies before their optical maxima. The Xinglong Station, at a latitude of 40.5 degrees, is about $900 \mathrm{~m}$ in altitude and 150 kilometer northeast of Beijing with no city-light pollution. The best observing seasons are winter and spring, which is ideal for SN searches because of the long nights and more nearby galaxies observable than other seasons. The $2.16 \mathrm{~m}$ telescope 
of BAO is conveniently located on the same mountain and can be used to get spectroscopy for SNe.

Modifications to the $60 \mathrm{~cm}$ telescope began in 1995 and continued until mid 1996. The optics was modified so that a CCD camera could be installed at the primary focus of the telescope. Software was compiled to automatically control the telescope and the CCD camera and to process the observations. Meanwhile a sample of about 5,000 nearby galaxies was constructed from the Third Reference Catalogue of Bright Galaxies (RC3, De Vaucouleurs 1991), the New General Catalogue of Galaxies (NGC, Huchra 1993), and the Uppsala General Catalogue of Galaxies (UGC, Warren 1993). Our strategy was to find as many $\mathrm{SNe}$ as possible, as early as possible. The system can observe about 500 galaxies in one night and recycle the sample in about one week.

BAOSS discovered the first SN, SN 1996W, in April of 1996, which is also the first SN discovered by Chinese astronomers with a telescope. Six SNe were discovered during the first year of operation (1996) and 17 were discovered in 1997. In four years, a total of 40 SNe were discovered by BAOSS, most of which well before their optical maxima. Some of these SNe were well observed and studied, both by BAO and by other astronomers worldwide. The success of this system proves that a small dedicated automatic telescope is the best way to do SN searches.

More modifications to the system were done in 2000. A CCD camera with a better quantum efficiency than the old one was purchased, and the optics of the telescope was modified so that the camera could be installed at the Cassegrain focus. These changes improved the efficiency for both SN searches and SN photometry. A small auto-guiding telescope was also installed which enabled us to take long exposures. More emphasis was put on finding SNe really early (e.g., two weeks before maxima), so the sample of galaxies was reduced to 2,500. With the current high efficiency each galaxy in the sample can be revisited every 3 to 4 days.

This paper is organized as follows. The hardware of the system is described in Section 2 and the software in Section 3. The discoveries are reported in Section 4. Section 5 discusses several well-observed SNe in BAOSS.

\section{The Telescope and the Detector}

The $60 \mathrm{~cm}$ telescope used by BAOSS has an $\mathrm{f} / 15$ Cassegrain system. The focal ratio at the prime focus is $\mathrm{f} / 4.3$. The telescope is automatically controlled by a PC.

The original CCD camera mounted at the primary focus was a TI 215 with $1024 \times 1024$ pixels. The pixel size was $12 \mu$, yielding a scale of about 1"/pixel. The field of view (FOV) of the camera was 17' X 17'. The camera was thermoelectrically cooled to about $-50^{\circ} \mathrm{C}$, so the dark current was relatively high. In 2000, we purchased a new CCD camera manufactured by Princeton Instruments, which is liquid nitrogen cooled and has a peak quantum efficiency of over $90 \%$. The new camera has $1340 \times 1300$ pixels, each $20 \mu \mathrm{m}$ in size. The FOV is reduced to 10' X 10' but it is still big enough for SN search and photometry observations. 


\section{The Software}

The software for BAOSS keeps evolving over time, but the main purpose remains the same: to schedule observations, to process the images automatically to find $\mathrm{SNe}$, and to automatically control the hardware.

\subsection{The Scheduling Program}

This program has two functions. One is to select objects from the master sample of galaxies to be observed for the night, and the other is to arrange for their observation at the most appropriate time. The weather condition and the moon phase are considered by the program in order to optimize the selection of the objects. During nights that are partly cloudy, the observer can opt to choose bright nearby galaxies.

The major selection criteria for the galaxies are their priorities, defined as follows:

$$
\text { Priority }=(D 1-D 0) / D
$$

where $D 1$ is the current date, $D 0$ is the date of the last observation, and $D$ is the control time.

The choice of the control time $D$ reflects our survey strategy. For galaxies between $20 \mathrm{Mpc}-100 \mathrm{Mpc}$, we choose $D$ to be as big as possible, as long as no $\mathrm{SNe}$ will be missed by the search. For galaxies less than $20 \mathrm{Mpc}$, we choose $D$ to be the duration of a normal SNe Ia between the limiting magnitude of our system and its peak magnitude in the corresponding galaxy. Assuming ideal weather and no competition, these choices of $D$ would enable our search to find all the nearby SNe before their maxima, and to find all the more distant SNe.

Once the priorities for all the galaxies are calculated, the program selects the galaxies from higher to lower priorities, with additional consideration of hour angles of the targets and the moon phases. This scheduling program is very useful for BAOSS. During seasons with few available observing nights, it is essential to use the scheduling program to make good use of the limited observation time.

\subsection{The Image Processing Program}

The principle of finding supernovae is to find new objects in the current observations by comparing them to the existing template images. A template image for a galaxy is the one with the highest quality, but if the new observation is found to be better than the template, the new image becomes the new template. Since SNe are likely to occur in the optical disks of the host galaxies, it is often difficult to find them visually because of the light contamination from the host galaxies. The goal of the image processing is thus to remove the complicated galaxy background and to reveal any changes in the images.

Since the new observation and the template are taken under different conditions (pointing, seeing, moon phase, transparency), various steps have to be taken before a final subtraction can be achieved. First, the two images are aligned to the same position, then convolved so the stars in the images have a similar Point-Spread-Function (PSF). Finally, the intensity levels of the images 
are matched. New objects can then be detected in the resultant subtraction and listed as SN candidates. These candidates are reobserved and if any of them are confirmed, reports are sent to the Central Bureau of Astronomical Telegrams (CBAT). If possible, spectroscopy is also attempted by the $2.16 \mathrm{~m}$ telescope.

The first version of the image processing program was compiled in 1995, based on programs provided by Brian Schmidt. It was heavily modified in the summer of 2000 using new tasks then available to IRAF (the Image Reduction and Analysis Facility) in the package "images/immatch".

An example is shown in Fig. 1 to explain how the program works to find SN 2000dw in UGC 11955. Four images are displayed: A) the new observation; B) the template image; C) the new observation after image alignment, PSF matching, and intensity matching; and D) the subtracted image with SN 2000dw standing out. It is obviously much easier to find SN $2000 \mathrm{dw}$ in image " $D$ " than in image " $\mathrm{A}$ ".

\subsection{The Automatic Observation Programs}

The automatic observation programs have two parts: one part controls the CCD camera and is written in Visual Basic Language; the other controls the telescope and is written in $\mathrm{C}$ Language. The two programs communicate with each other via a Linux network. The automatic observations are taken in the following sequences. The telescope is first pointed to the new target and if at position, the CCD camera is directed to start an exposure. When the exposure is finished the telescope moves to the next object and the cycle continues.

\section{The Discoveries}

The first supernova discovered by BAOSS was SN 1996W (Li et al. 1996). From 1996 April to $2001 \mathrm{March}, 40 \mathrm{SNe}, 5$ cataclysmic variables, and 2 extra-galactic novae have been discovered by BAOSS. A brief listing of the SNe statistics discovered by BAOSS is presented in Table 1. Table 2 lists more details of these $\mathrm{SNe}$.

Table 1. Statistics of the SNe discovered by BAOSS

\begin{tabular}{|c|c|c|c|c|c|}
\hline \hline Total & Ia(normal) & Ia(peculiar) & II(normal) & II(peculiar) & Ib \&Ic \\
\hline 40 & 10 & 4 & 18 & 3 & 5 \\
\hline
\end{tabular}

In Table 1, "Ia(normal)" are those SNe Ia that show normal spectra like SN 1986I. "Ia(peculiar)" are those SNe Ia that are similar to either 1991T (overluminous) or 1991bg (subluminous). "II(normal)" are those Type IIP and IIL SNe. "II(peculiar)" are those Type IIn and IIb SNe.

In the BAOSS sample, SNe II represent about 50 percent of the total, SNe Ia 35 percent, and $\mathrm{Ib} / \mathrm{Ic}$ about 12 percent. This frequency distribution is different from that found by Cappellaro et al (1999). In particular, the percentage of $\mathrm{SNe}$ Ia in the BAOSS sample seems to be lower than theirs. One possible explanation is that only $\mathrm{SNe}$ Ia occur in both spiral and elliptical galaxies, and a possible bias against elliptical galaxies in the BAOSS galaxy sample may have resulted 


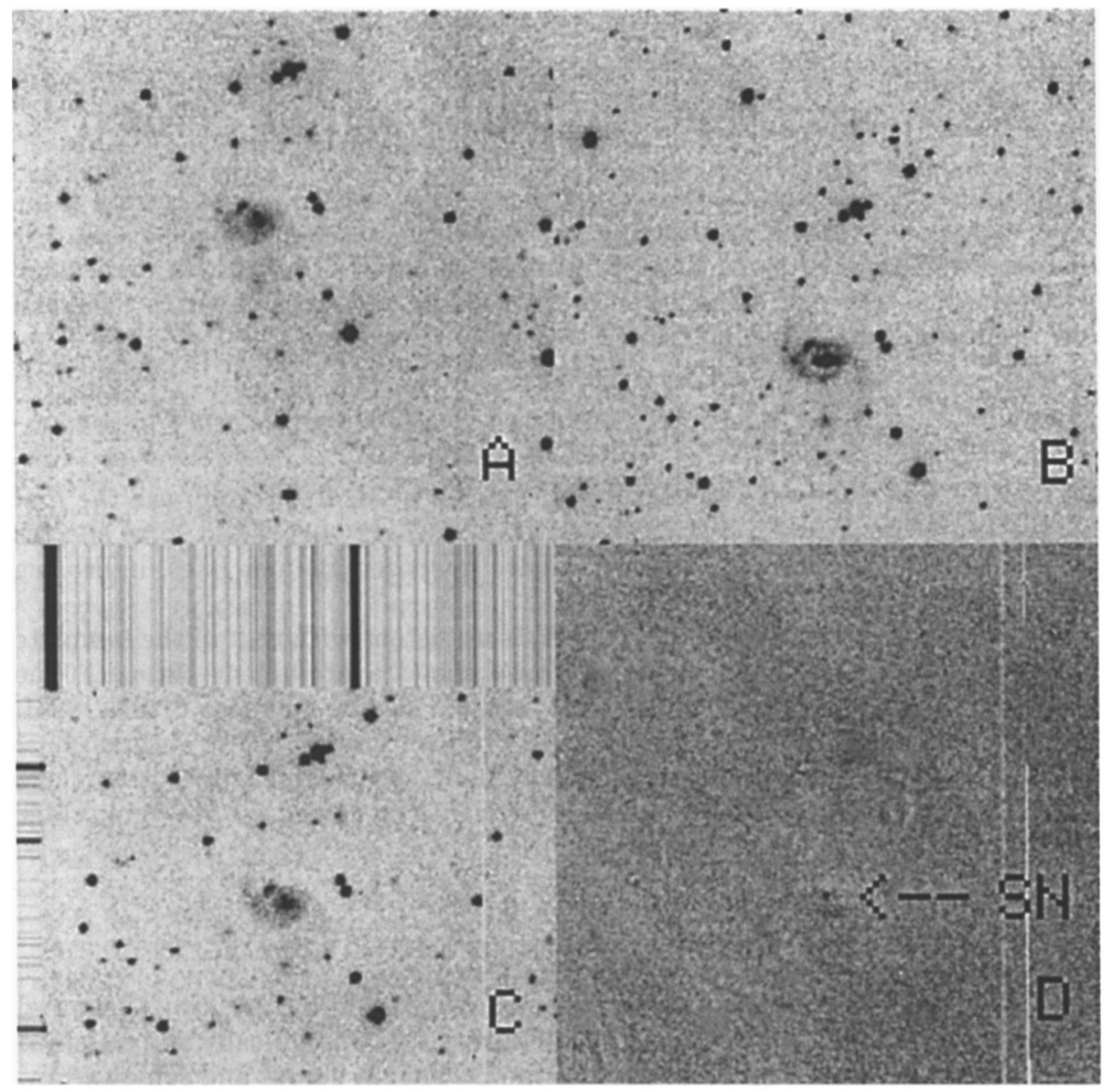

Figure 1. Example images of the supernova finding program. A: the new observation; B: the template image; $\mathrm{C}$ : the new observation after image alignment, PSF matching, and intensity matching; D: the subtracted image with SN $2000 \mathrm{dw}$ identified. 
in a smaller percentage of SNe Ia. The low SN Ia frequency could, however, be caused by the small number statistics.

One interesting result of the statistics from our discoveries is the high occurence rate of peculiar SNe Ia. Combining our discoveries with those of the Lick Observatory Supernova Survey (LOSS)(Li et al 2001), we found the rate of peculiar SNe Ia to be about $36 \% \pm 9 \%$, much higher than earlier estimates $(<17 \%$, Branch et al, 1993).

\section{Some Well Studied SNe Discovered by BAOSS}

One important goal of BAOSS is to do photometric and spectroscopic follow-up observations of bright SNe. Here we report on the observations of several SNe discovered in the course of BAOSS. The photometric observations were taken with the $60 \mathrm{~cm}$ telescope and the spectroscopic date were obtained with the OMR low resolution spectrograph of the $2.16 \mathrm{~m}$ BAO telescope.

\subsection{SN $1996 \mathrm{~W}$ in $\mathrm{NGC4027}$}

SN 1996W was discovered on April 10, 1996 (Li et al. 1996) and was the first SN discovered by BAOSS. Fig. 2 shows the BVR light curves and some spectra of SN 1996W. The spectra show prominent hydrogen Balmer lines, indicating that SN $1996 \mathrm{~W}$ is a Type II SN.

The B band light curve of SN $1996 \mathrm{~W}$ is consistent with that of the template Type IIP SN. The V band light curve also shows a prominent plateau. The spectra show typical Type IIP spectral features similar to those of SN 1986I. In particular, the $\mathrm{H} \alpha$ shows a conspicuous $\mathrm{P}$ Cygni profile. These observational features are consistent with the suggestion made by Schlegel (1996), that there is a correlation between the plateau shape of light curves and the P Cygni profile of the spectra of Type IIP supernovae.

\subsection{SN 1996cb in NGC3150}

SN 1996cb was discovered by Japanese astronomers and by BAOSS (Qiao et al 1996) independently. We confirmed the discovery spectroscopically and classified it as a Type II SN. In Fig. 3, we present the BVR light curves and some spectra of SN $1996 \mathrm{cb}$.

The first few spectra of SN 1996cb showed that it was a typical type II, with prominent hydrogen Balmer lines. But as the supernova evolved, the spectra deviated from typical type II SNe and became similar to those of type Ib supernovae. These kind of supernovae are defined as Type IIb SNe (Wheeler \& Filippenko 1996, Filippenko 1996). SN 1996cb turned out to be the third type IIb SN ever discovered, the other two being SN 1987K and SN 1993J.

The light curves of SN 1996cb are very similar to those of SN 1993J. SN $1996 \mathrm{cb}$ resembles SN 1993J in its spectral evolution except for some subtle differences (Qiu et al 1999), from which we concluded that the progenitor of SN $1996 \mathrm{cb}$ was smaller than that of SN $1993 \mathrm{~J}$ but has a thicker hydrogen envelop. 
Table 2. Supernovae Discovered By the BAO Supernova Survey

\begin{tabular}{|c|c|c|c|c|c|}
\hline$\overline{\mathrm{SN}}$ & Galaxy & Coordinates of SN & Mag & $\begin{array}{l}\text { SN } \\
\text { Type }\end{array}$ & $\begin{array}{r}\text { Discovery } \\
\text { Date }\end{array}$ \\
\hline $1996 \mathrm{~W}$ & NGC4027 & $115928.98-191521.9$ & $\overline{V 16.0}$ & II & $1996 \mathrm{Apr} 10$ \\
\hline 1996 bo & NGC673 & $014822.86+113115.1$ & V16.5 & Ia & Oct 18 \\
\hline 1996 bv & UGC3432 & $061613.04+570308.4$ & V16.6 & Ia & Nov 3 \\
\hline $1996 \mathrm{bw}$ & NGC664 & $014344.51+041319.0$ & 17.5 & II & Nov 30 \\
\hline 1996 by & UGC3379 & $055824.96+682712.1$ & 16.5 & Ia & Dec 14 \\
\hline $1996 \mathrm{cb}$ & NGC3510 & $110341.98+285413.7$ & 16.5 & IIb & Dec 15 \\
\hline $1997 \mathrm{C}$ & NGC3160 & $101356.18+384900.5$ & 17.5 & Ic & $1997 \mathrm{Jan} 13$ \\
\hline $1997 \mathrm{Y}$ & NGC4675 & $124531.40+544417.0$ & 14.8 & Ia & Feb 2 \\
\hline $1997 \mathrm{aa}$ & IC2102 & $045153.60-045736.0$ & 17.0 & II & Mar 1 \\
\hline $1997 \mathrm{bn}$ & UGC4329 & $081902.20+211101.7$ & 16.4 & II & Apr 3 \\
\hline 1997 bo & A $121952+0743$ & $121952.66+074349.7$ & 16.1 & II & Apr 3 \\
\hline $1997 \mathrm{br}$ & ESO576-G40 & $132042.40-220212.3$ & 14.5 & Iapec & Apr 10 \\
\hline $1997 \mathrm{cn}$ & NGC5490 & $140957.76+173232.3$ & 17.2 & Iapec & May 19 \\
\hline 1997 co & NGC5125 & $132401.18+094222.9$ & 18.2 & II & May 29 \\
\hline $1997 \mathrm{cw}$ & NGC105 & $002517.27+125306.2$ & 16.5 & Iapec & Jul 10 \\
\hline $1997 \mathrm{dc}$ & NGC7678 & $232828.41+222523.0$ & 18.3 & $\mathrm{Ib}$ & Aug 5 \\
\hline $1997 \mathrm{dg}$ & A234014+2612 & $234014.21+261211.8$ & 16.7 & Ia & Sep 27 \\
\hline $1997 \mathrm{di}$ & UGC4015 & $074744.45+621939.2$ & 18.1 & II & Oct 27 \\
\hline $1997 \mathrm{do}$ & UGC3845 & $072642.50+470536.0$ & 15.8 & $\mathrm{Ia}$ & Oct 31 \\
\hline $1997 \mathrm{ds}$ & M-01-57-007 & $222411.51-032910.5$ & 16.2 & II & Nov 17 \\
\hline $1997 \mathrm{dt}$ & NGC7448 & $230002.93+155850.9$ & 15.4 & $\mathrm{Ia}$ & Nov 21 \\
\hline $1998 \mathrm{C}$ & UGC3825 & $072334.63+412604.2$ & 18.1 & II & 1998 Jan 21 \\
\hline $1998 \mathrm{D}$ & NGC5440 & $140259.28+344454.3$ & 15.5 & Ia & Jan 29 \\
\hline $1998 \mathrm{~S}$ & NGC3877 & $114606.11+472855.8$ & V16.0 & IIpec & Mar 2 \\
\hline $1998 \mathrm{~T}$ & NGC3690 & $112833.16+583343.7$ & 14.5 & $\mathrm{Ib}$ & Mar 3 \\
\hline $1998 \mathrm{ab}$ & NGC4704 & $124847.24+415528.3$ & B16.6 & Iapec & Apr 1 \\
\hline 1998 ar & NGC2916 & $093458.86+214257.5$ & 18.4 & II & Apr 14 \\
\hline $1998 \mathrm{dn}$ & NGC337A & $010127.08-073636.7$ & 15.8 & II & Aug 22 \\
\hline $1998 \mathrm{ec}$ & UGC3576 & $065306.11+500222.1$ & 16.9 & Ia & Sept 26 \\
\hline $1999 \mathrm{D}$ & NGC3690 & $112829.50+583340.6$ & 15.6 & II & 1999 Jan 16 \\
\hline 1999 аа & NGC2595 & $082741.11+212915.5$ & 16.3 & Ia & Feb 11 \\
\hline 1999 an & IC755 & $120110.5+14061$ & 15.0 & II & $\operatorname{Mar} 7$ \\
\hline $1999 \mathrm{dn}$ & NGC7714 & $233614.70+020908.8$ & 16.0 & Ib & Aug 19 \\
\hline 1999 ed & UGC3555 & $065000.95+253754.5$ & 17.8 & II & OCt 5 \\
\hline 1999 el & NGC6591 & $203717.83+660611.5$ & 15.4 & IIn & Oct 19 \\
\hline $2000 \mathrm{dw}$ & UGC11955 & $221349.06+391415.1$ & 17.5 & II & 2000 Oct 17 \\
\hline $2001 \mathrm{~B}$ & IC 391 & $045719.24+781116.5$ & 15.5 & $\mathrm{Ib}$ & 2001 Jan 3 \\
\hline $2001 \mathrm{~K}$ & IC 677 & 111356.09121806 .6 & 17.8 & II & Jan 15 \\
\hline $2001 \mathrm{~T}$ & MCG-02-37-6 & $143253.17-125843.6$ & 16.8 & II & Feb 6 \\
\hline $2001 X$ & NGC 5921 & $152155.45+050342.1$ & 17.0 & II & Feb 27 \\
\hline
\end{tabular}


Light Curves of SN 1896W

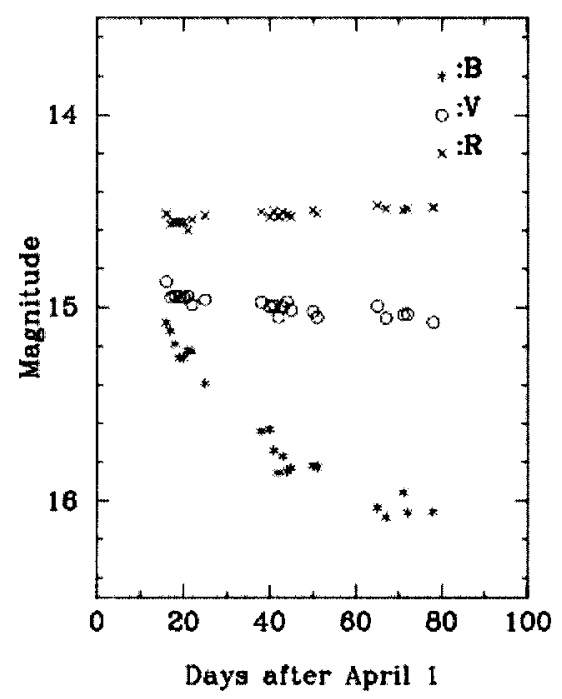

Spectra of SN 1996 W

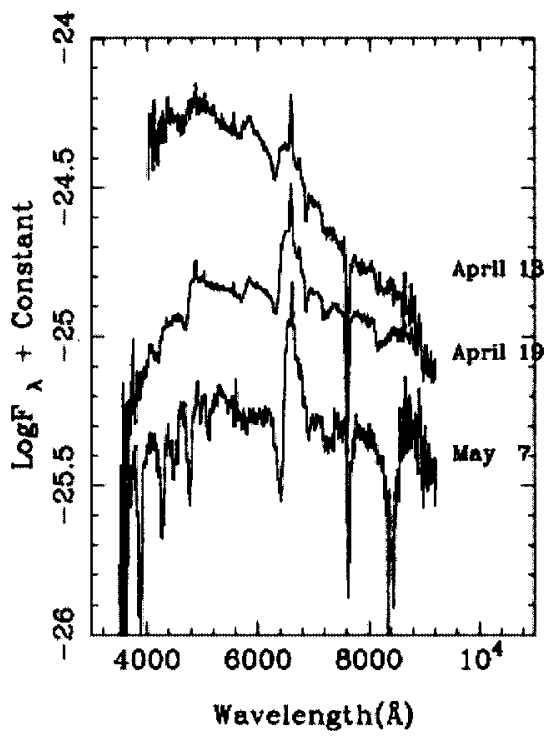

Figure 2. The light curves and spectra of SN $1996 \mathrm{~W}$

Light Curves of SN $1996 \mathrm{cb}$

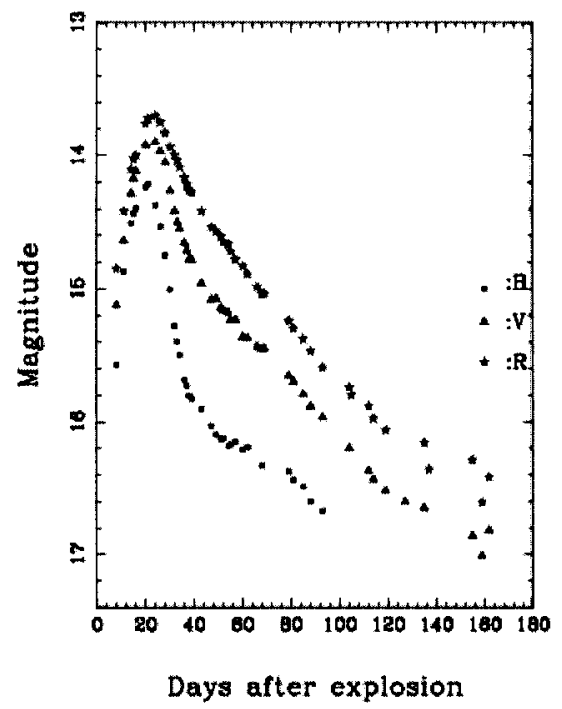

Spectra of SN $1896 \mathrm{cb}$

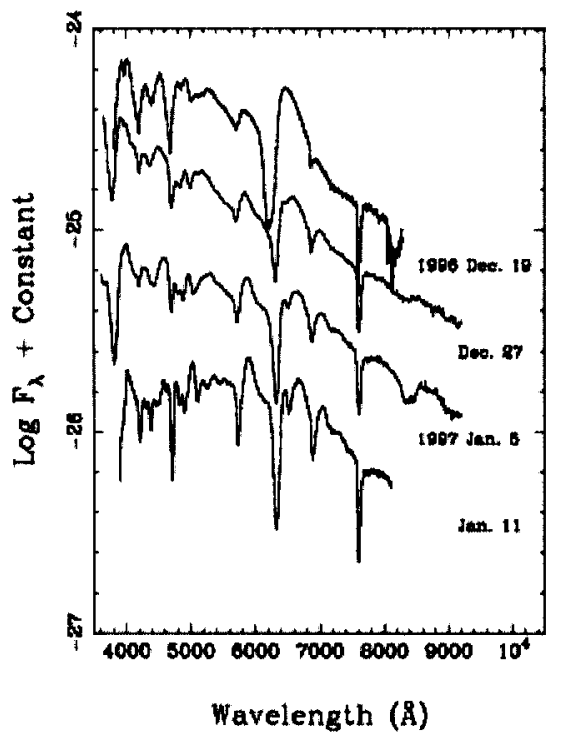

Figure 3. The light curves and spectra of SN $1996 \mathrm{cb}$ 
Light Curves of SN $1997 \mathrm{br}$

Spectra of SN 1997br
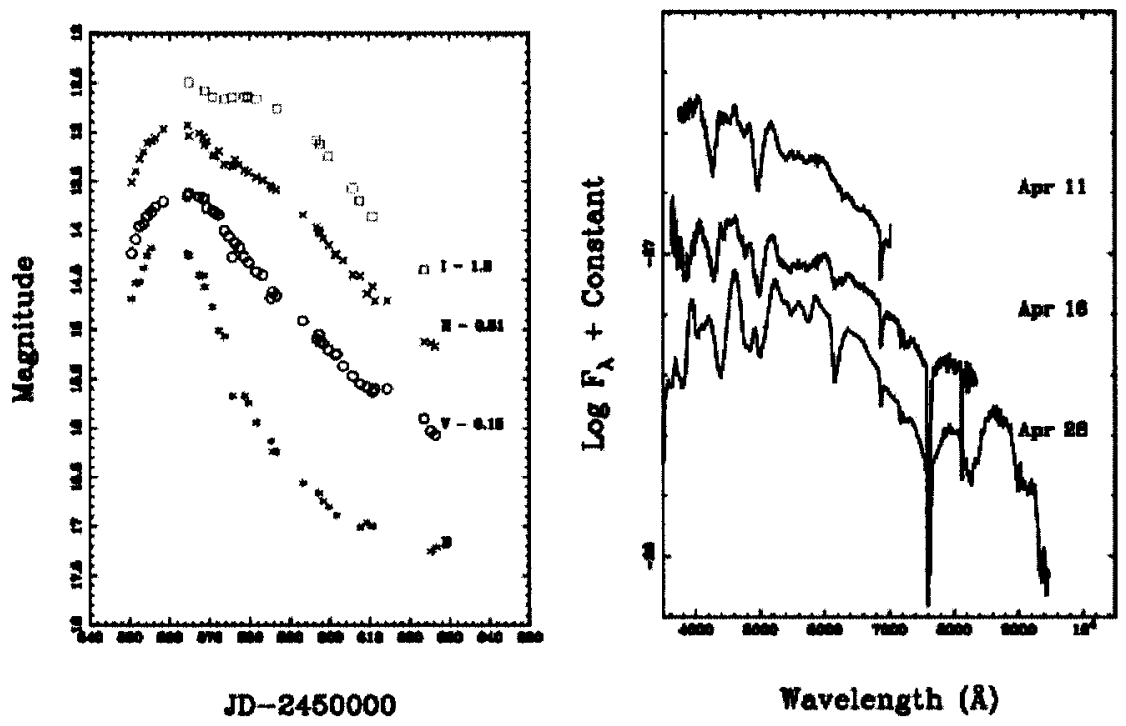

Figure 4. The light curves and spectra of SN $1997 \mathrm{br}$

\subsection{SN $1997 \mathrm{br}$ in ESO $576-\mathrm{G} 40$}

SN 1997br was discovered by BAOSS on Apr. 10.6 UT, 1997 (Li et al 1997). In Fig. 4, we show the UBVI light curves and some spectra of SN 1997br.

SN 1997br is a peculiar SN Ia whose prototype is SN 1991T (Filippenko et al 1992). The spectra of this kind of SNe Ia before maxima are dominated by high excitation Fe III lines. As the SN evolves, it develops lines of the intermediate-mass elements that are typical in normal SNe Ia.

The light curves of SN 1997br are similar to those of SN 1991T, an overluminous Ia SN, with a slow decline rate after the B maximum. The spectra of SN $1997 \mathrm{br}$ also resemble those of SN 1991T with subtle differences. Spectra of SN $1997 \mathrm{br}$ seem to indicate an earlier transition to the dominant phase of Fe-peak elements after the B maximum ( $\mathrm{Li}$ et al 1999).

\subsection{SN 1998S in NGC3877}

SN 1998S was discovered on Mar 2, 1998. It is a peculiar type II SN, showing narrow Balmer lines in the spectra. Schlegel (1990) defined this kind of SNe as IIn SNe, where " $n$ " stands for narrow lines. In Fig. 5, we present the BVR light curves and some spectra of SN 1998S.

SN 1998 S is a very bright SN reaching a magnitude of about 12 at maximum. It was discovered at a very early phase when it was only 16 mag in the $\mathrm{R}$ band. The absolute magnitude in the B band at the maximum for SN $1998 \mathrm{~S}$ is -18.6 , about 2 magnitude brighter than for a normal type II supernovae. Due to its brightness and peculiarity, SN 1998S has been extensively observed worldwide, including several visits by the Hubble Space Telescope. The spectral evolution 
Light Curves of SN 1898 S

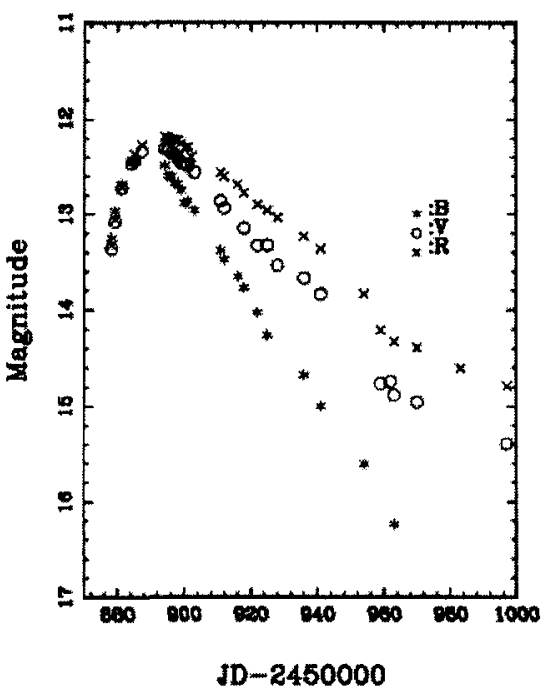

Spectra of SN $1898 \mathrm{~S}$

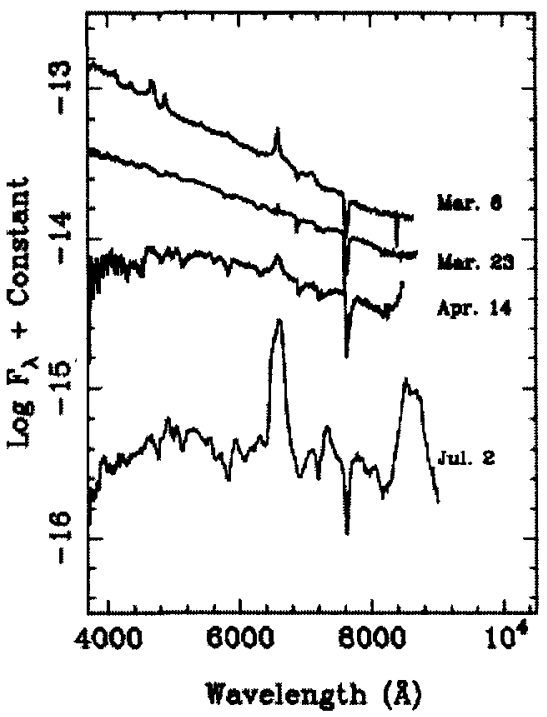

Figure 5. The light curves and spectra of SN 1998S

of SN 1998S (Liu et al 2000) is similar to that of SN 1979C, a typical supernova of SNe II-L. The spectra before maximum showed high-ionization N III and He II emission lines superimposed on a strong blue continuum, which is the WolfRayet star feature. The light curves showed a steep and linear decline, consistent with those of SN 1979C. Observations by HST and in near-infrared wavelenghts show that there is a strong interaction between the ejecta and the circumstellar materials of the SN. SN 1998S thus provides a good opportunity to study the environment of SNe.

Acknowledgments. BAOSS is supported by the National Science Foundation of China. We would like to thank the support from one sub-project of the 973 Project, "NKBRSF G 19990754".

\section{References}

Branch, D., Fisher, A., Nugent, P. 1993, AJ, 106, 2338

Cappellaro, E., Evans, R., Turatto, M. 1999, A\&A, 351, 459

De Vaucouleurs, A., Corwin, H. G. 1991, Third Reference Catalogue of Bright Galaxies (New York: Springer-Verlag)

Filippenko, A. V., Richmond, M., Matheson, T. et al. 1992, ApJ, 384, L15

Filippenko, A. V. 1997, in Thermonuclear Supernovae, ed. R. Canal, O. RuizLapuente, \& I. Isern (Dorfrecht: Kluwer), 1

Huchra, J. P. 1993, Redshifts Catalogue, Electronic version, Washington D. C.: NASA, 
Li, W. D., Qiu, Y. L., Zhu, X. H.; Hu, J. Y. 1999, AJ, 117, 2709

Li, W., Qiao, Q., Qiu, Y., Hu, J. 1996, IAUC 6497

Li, W. \& Li C. 1998, IAUC 6829

Li, W., Filippenko, Treffers, R., Riess, A., Hu, J., Qiu, Y. 2001, ApJ, 546, 734

Liu, Q. Z., Hu, J. Y., Hang, H. R.; Qiu, Y. L., Zhu, Z. X., Qiao, Q. Y. 2000 A\&AS, 144, 219

Perlmutter, S., Aldering, G., Goldhaber, G. et al 1999, ApJ, 517, 565

Qiao, Q., Li, W., Qiu, Y., Hu, J. 1996, IAUC 6527

Qiu, Y., Li, W., Qiu, Q., Hu, J. 1999, AJ, 117, 736

Schlegel, E. M., 1990, MNRAS, 244, 269

Schlegel, E. M. 1996, AJ, 111, 269

Schmidt, B., Suntzeff, N., Phillips, M. et al 1998, ApJ, 507, 46

Warren, W., H., Uppsala Catalogue of Galaxies, Electronic version, Washington D. C.: NASA, 1993

Wang, L.F., \& Hu, J. Y. 1994, Nature, 369, 380

Wheeler J. C., \& Filippenko, A. V. 1996, in IAU Colloq. 145, Supernovae and Supernova Remnants, Ed. R. McCray \& Z. Wang(New York: Cambridge Univ. Press), 241

Wheeler, J. C., Yi, I., Hoflich, P., \& Wang, L. 2000, ApJ, 537, 810 
